\title{
RECHT ALS PRAXIS
}

\author{
Meinrad Handstanger
}

Abstract: Die Perspektive der Rechtsanwendung ist für die Fixierung des Norminhaltes von fundamentaler Bedeutung. Der Inhalt einer konkreten Norm richtet sich prinzipiell nach ihrer Verwendung im juristischen Gebrauchskontext. Juristische Richtigkeit basiert auf dem Verwendungserfolg von Argumenten. Die Rechtsprechung weist inhaltliche Diskontinuitäten auf, die mit Legal Tech nicht hinreichend prognostiziert werden können. Zur Unterstützung der juristischen Entscheidungsfindung und -textierung wäre eine termorientierte Aufbereitung von Rechtssätzen wünschenswert.

\section{Inhaltsverzeichnis}

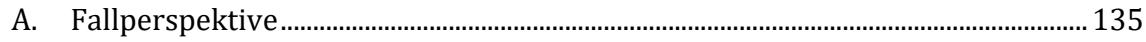

B. Duales Wahrheitskonzept.........................................................................................137

C. Fallibilität und Falsifizierbarkeit .................................................................................. 138

D. Norminhalt und Rechtspraxis .................................................................................... 139

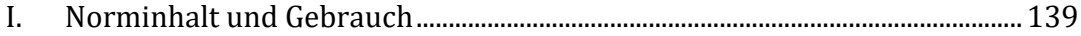

II. Judikatur und Gesetzesinhalt ................................................................................ 140

E. Kontinuität und Diskontinuität ......................................................................................... 143

F. Die Mesoebene der Rechtssätze ........................................................................................ 144

\section{A. FALLPERSPEKTIVE}

Rechtsdogmatik beginnt - so JOACHIM LEGE in einer rechtsrealistischen Betrachtung «in Wahrheit mit dem zweiten Fall, dem Vergleichsfall». Das Recht hat in Wahrheit «seine Realität im konkreten Fall, der entschieden werden muss»1.

1 LEGE, Was Juristen wirklich tun. Jurisprudential Realism, in: BRUGGER/NEUMANN/KIRSTE (Hrsg), Rechtsphilosophie im 21. Jahhundert (2008) 207, 208, 224 ff. 
Dem kann man wohl nicht wirksam entgegentreten, weil die Perspektive des Falles und der Falllösung den Umgang mit Rechtsvorschriften, darauf gestützten Fallentscheidungen, aber auch der - rechtsdogmatischen - systematischen Beschreibung des Inhaltes der Rechtsvorschriften maßgeblich prägt.

Sowohl rechtswissenschaftliche Lehre als auch Judikatur versuchen, den von Rechtsnormen jeweils erfassten Bereich mit ihrem Regelungsinhalt festzumachen und beziehen sich derart auf die Konstellationen, die sachverhaltsmäßig von einer Rechtsvorschrift abgedeckt werden. Freilich scheint ihre Annäherung gegenläufig: Während in der Judikatur ganz konkrete Fälle zu entscheiden sind, lässt sich die Lehre bei der Beschreibung des Norminhalts grundsätzlich von einer typisierenden Betrachtung der möglichen Fälle leiten, die unter die Norm fallen werden. Lehre reflektiert dabei allerdings regelmäßig schon bestehende Judikatur, ebenso wie Judikatur grundsätzlich zur Interpretation die von der rechtsdogmatischen Lehre erarbeiteten Argumente heranzieht und mit der Entwicklung von Judikaturlinien ihrerseits wiederum zu einer typologischen und systemischen Betrachtung gelangt, die sie häufig auch systematisierend aufzeigt. Rechtsdogmatik wird damit jedenfalls auf der Ebene der gerichtlichen Grenzorgane, bei denen die gerichtlichen Kontrollzüge enden und die bestehenden Rechtsprechungslinien in concreto durchsetzbar sind, zu einem gemeinsamen Format von Lehre und Rechtsprechung. Ausgehend davon sind beide letztlich (auch) fallorientiert. Die «Teilnehmerperspektive» (üblicherweise bei der juristischen Praxis verortet) und die «Beobachterperspektive» ${ }^{2}$ (die v.a. die universitäre Dogmatik repräsentieren soll) lassen sich nicht trennscharf voneinander abgrenzen; jede Teilnahme verlangt zur Feststellung der Rechtslage die Beobachtung der fallspezifisch einschlägigen Lehre und Rechtsprechung, jede außenstehende Beobachtung kann ohne die teilnehmerperspektivisch relevanten (vertypten) Fallkonstellationen den Inhalt einer Rechtsnorm nur oberflächlich beschreiben. Auch von Rechtsnormen Verpflichtete werden sich an Judikatur und Lehre orientieren, zumal sie prinzipiell daran interessiert sein werden, nicht in den Fokus der behördlichen Rechtsanwendung zu geraten.

2 Zu diesen Perspektiven der Rechtsbetrachtung vgl. KoLLER, Theorie des Rechts (1997) 47 ff, insb 49. 


\section{B. DUALES WAHRHEITSKONZEPT}

Die Frage, ob Sachverhalte tatsächlich vorliegen, um darauf bezogene Rechtsnormen zum Tragen zu bringen, verlangt eine Analyse der konkreten Sachlage, zu der in der Regel besondere Kenntnisse des Sachbereiches notwendig sind. Der sachverstandsmäßige Input muss aus den Wissens- bzw. Wissenschaftsbereichen erfolgen, die diese Analyse erbringen können. Damit werden für das rechtliche Verfahren die auch dort maßgebenden Wahrheitskonzepte bedeutsam, welche oft naturwissenschaftlich geprägt sind.

Aussagen über den Inhalt bzw. die rechtliche Bedeutung von Rechtsnormen folgen - so wird das überwiegend gesehen - demgegenüber (bloß) einem Konzept der Richtigkeit. Ausgehend davon, dass Rechtsnormen nach den Auslegungsstandards sehr oft mehrere Verständnisvarianten eröffnen, geht es um die Identifikation der richtigen Alternative. Rechtsprechung und Lehre richten sich dabei nach einem wertenden Vergleich: richtig erscheint jene Alternative («Normhypothese»), für die (derzeit) die besseren Gründe sprechen. Die Überzeugungskraft - Plausibilität - dieser Normhypothese übertrifft die der übrigen.

Schlüsselbegriff dieses Ansatzes ist das Argument. Argumente werden hier verstanden als die Gründe, die für bzw. gegen die Plausibilität einer Normhypothese sprechen. Aus den Falllösungen der Gerichte und den Darstellungen der (akademischen) Lehre lassen sich solche Argumente gewinnen.

Bei der Suche nach den besseren Gründen geht es daher um die Analyse dieser Bereiche dahin, welche Argumente überhaupt in Betracht kommen und welche Erfolg dahin hatten, als die besseren identifiziert zu werden. Die Richtigkeit der Beschreibung des Inhaltes - Sinnes - einer Rechtsvorschrift orientiert sich an diesen besseren Argumenten. Der «zwanglose Zwang» der Überzeugungskraft ergibt sich aus dem Inhalt, aber auch aus der Position desjenigen, der dieses Argument verwendet. Etwa von gerichtlichen Grenzorganen geteilte Argumente haben grundsätzlich eine höhere Erfolgsaussicht, dort in neuen letztinstanzlichen Verfahren wiederum durchzudringen, als von diesen Organen nicht geteilte Argumente.

Die nach Wahrheitskonzepten anderer Wissenschaften erarbeiteten (meist gutachterlichen) Verfahrensbeiträge werden nach den dafür relevanten verfahrensrechtlichen 
Rechtsvorschriften im Verfahren berücksichtigt, was eine Art «Überwölbung» dieser Beiträge auf Basis des juristischen Richtigkeitskonzepts bedeutet.

\section{FALLIBILITÄT UND FALSIFIZIERBARKEIT}

Auf dieser Grundlage richtet sich juristische «Wahrheit» als Plausibilität nach der Überzeugungskraft und damit letztlich nach dem Verwendungserfolg von Argumenten.

Der Verwendungserfolg ist freilich kontextabhängig. Selbst wenn man von weitgehend gleichförmig konstellierten juristischen Diskurs- bzw. Verfahrenskontexten ausgeht, kann sich doch im Lauf der Zeit (gerade im Instanzenzug durch übergeordnete Gerichte) die Plausibilität von Argumenten und des von ihnen getragenen Normverständnisses ändern. Mit anderen Worten bedeutet das, dass juristische Auslegungsergebnisse grundsätzlich fehlbar und gerade durch spätere Auslegungen falsifizierbar ${ }^{3}$ sind. Dies gilt auch für höchstgerichtliche Rechtsprechung, etwa wegen relevanter Sach- und Rechtsänderungen oder neue, überzeugendere Argumentationen ${ }^{4}$. Zu Grunde liegen solchen Änderungen Lernprozesse, die ihrerseits Standards juristischen Arbeitens folgen.

Letztkriterien juristischer Richtigkeit (eine Rechtsidee, Gerechtigkeit oder ähnliches) sind für dieses Plausibilitätskonzept weder erforderlich noch generell überzeugend 5 festmachbar. Vielmehr kommt der juristischen Richtigkeit ein Eigenwert zu${ }^{6}$, der sie inhaltlich von anderen Bereichen (etwa Ethik oder Moral) absetzt. Außerdem ist das juristische Geschehen derart systemisch autonom, dass die (etwa durch richterliche Garantien) unabhängige Produktion seiner Entscheidungen und Beurteilungen gesichert ist, damit es seine Funktion im gesellschaftlichen bzw. politischen Gesamtkontext erfüllen kann. Dies ist nicht nur für pluralistisch verfasste politische Systeme bedeutsam7,

3 Vgl dazu etwa Heinz von Foerster, Kybernetische Reflexionen, in: FisCher/RetZer/Schweitzer (Hrsg), Das Ende der großen Entwürfe, 132.133 f.

4 Etwa im Zuge der Etablierung einer neuen herrschenden Lehre.

5 Vgl idZ KeLSEN, Was ist Gerechtigkeit? (1953)

6 Vgl LEGE, Pragmatismus und Jurisprudenz (1999) 562.

7 «Rechte machen Leute», vgl. LESSENICH, Grenzen der Demokratie (2019) 25. 
für letztere aber fundamental, als dort das Recht die generell geteilte Grundlage des Zusammenlebens absichert und durchsetzbar macht.

\section{NORMINHALT UND RECHTSPRAXIS}

\section{Norminhalt und Gebrauch}

Der Inhalt einer konkreten Norm richtet sich damit nach ihrer Verwendung im juristischen Gebrauchskontext, zu dem hier auch eine akademische Aufbereitung gezählt wird, die die in Normen vertypten Fallkonstellationen behandelt. Der Norminhalt wird bei der Normanwendung festgelegt.

Normdeutungen und die damit zusammenhängenden Argumente, die sich in der juristischen Meinungsbildung bewähren, determinieren diesen Gebrauchskontext. Letztlich sind das v.a. jene Deutungen, die sich rechtsschutzmäßig durchsetzen lassen. Dass solche Deutungen mitunter aus rechtlicher Perspektive trefflich in Kritik gezogen werden können, zeigt die rechtswissenschaftliche Analyse von Judikatur, die ihrerseits zu Deutungsänderungen führen kann, die sich wiederum bewähren.

Damit lässt sich freilich behaupten, dass eine andere als die bewährte Deutung die in Wahrheit eigentlich zutreffende sei. Eine solche andere Auffassung beleibt unbenommen, hat sich aber alleine mit ihrer Geltendmachung noch nicht bewährt, weshalb sie (noch) keine Richtigkeit im Sinn der bisherigen Überlegungen beanspruchen kann.

Da sich keine Norm selbst zu deuten fähig ist, sondern vielmehr zur Feststellung ihres Inhalts einen deutende, die Norm interpretierende Person verlangt, kann der Norminhalt nur im Wege darauf gerichteter Aktivität wahrgenommen werden.

Diese Aktivität ist (wie angesprochen) darauf gerichtet, jene Argumente bzw. Kombination von Argumenten ${ }^{8}$ zu identifizieren, die im Sinn der Logik des besseren Arguments zum besten Deutungsergebnis führen. Diese argumentative Aktivität reflektiert

8 Vgl. idZ etwa FIEDLER, Richterliche Rechtsanwendung als Modellbildungsprozesse, in: SCHWEIGHOFER/LieBWALD/AugENEDER/MENZEL (Hrsg), Effizienz von e-Lösungen in Staat und Gesellschaft - IRIS 2005 (2005) 45. 
insbesondere den zukünftigen Nutzen einer Argumentationsweise für weitere Rechtsanwendungsfälle. Insofern ist sie nicht nur vorgaben- und damit vergangenheitsorientiert, sondern auch zukunftsorientiert ${ }^{9}$.

\section{Judikatur und Gesetzesinhalt}

Die Beachtung der Rechtsprechung in diesem Sinn der Bewährung bringt eine der Rechtsanwendung generell inhärente Struktur zum Ausdruck ${ }^{10}$. Rechtsvorschriften mit gesetzlichem Charakter sind generelle Rechtsquellen. Einen generellen Charakter weist ihr Inhalt aber nur dann auf, wenn dieser für Fälle und Personen in gleicher Weise zum Tragen kommt.

Da Fallentscheidungen für bestimmte Sachverhaltskonstellationen den Inhalt der dafür einschlägigen Rechtsnormen konkretisieren, lässt die Nichtbeachtung einer schon vorhandenen Entscheidung in einem neuen Fall den generellen Charakter des Gesetzesinhaltes außer Acht. Der Inhalt einer schon vorhandenen Fallentscheidung ist nämlich nicht getrennt von den gesetzlichen Vorschriften denkbar, auf die sie sich stützt. Umgekehrt ist der Inhalt des Gesetzes von der in einer Entscheidung vorgenommenen Konkretisierung des Gesetzes für eine bestimmte Sachverhaltskonstellation nicht trennbar. Wenn neuerlich eine solche Konstellation zur Entscheidung ansteht, soll der Charakter des Gesetzes als generelle Norm gewahrt werden. Dies kommt jedenfalls für gefestigte höchstgerichtliche Judikatur zum Tragen. Im Fall der Nichtbeachtung dieser Rechtsprechung in einem neuen Fall kann nämlich (wie schon angesprochen) beim Höchstgericht der durch die gefestigte Judikatur determinierte Norminhalt durchgesetzt werden.

9 RICHARD A. POSNER bezieht die Folgenabschätzung nicht auf den argumentativen Kontext, sondern die tatsächlichen Entscheidungsfolgen, vgl. etwa DERS., Pragmatic Abjudication, in: TAlisSE/Aikin (Hrsg), The Pragmatism Reader (2011) 423, 425 ff. Dieser Ansatz tritt dort in den Hintergrund, wo - wie in kontinentalen Rechtssystemen Europas - diese Einschätzung in erster Linie dem Gesetzgeber selbst zukommt. Aber auch dort können die tatsächlichen Entscheidungsfolgen in die Logik des besseren Arguments einfließen. 
Eine Fallentscheidung versteinert aber den Norminhalt nicht. In der neu anstehenden Entscheidung kann davon infolge einer Neubewertung der sachlichen bzw. der rechtlichen Grundlagen abgewichen werden. Eine schon vorhandene Fallentscheidung bildet damit eine Grundlage für die Neubewertung und kommt nur bis dahin - «so far» - zum Tragen, während ab der Neubewertung - «from now on» - die neu getroffene Entscheidung für neue zu entscheidende Fälle maßgeblich ist, wie dies in Anlehnung an den Philosophen JOSEF MITTERER gesagt werden kann, der seine Überlegungen freilich aus einer ganz anderen erkenntnisleitenden Perspektive entwickelt hat ${ }^{11}$.

Der Norminhalt ergibt sich nicht derart ausschließlich aus dem Gesetzestext, sondern auch aus den danach getroffenen Fallentscheidungen. In diese Richtung weist die «authentische Auslegung» eines schon erlassenen Gesetzes durch den Gesetzgeber in der Form eines Gesetzes, die eine Änderung der Rechtslage bewirkt ${ }^{12}$. Mit einer authentischen Auslegung wird in der Regel das Verständnis korrigiert, das das Gesetz bisher in der Rechtsprechung gefunden hat, was die inhaltliche Konnexität von Gesetz und Gesetzesanwendung unterstreicht.

Fallentscheidungen stehen als vorläufiges Zwischenergebnis der Auslegungsgeschichte des Gesetzestextes wiederum (wie angesprochen) unter einer Art Falsifikationsvorbehalt. Erfolgt eine Neubewertung bestimmter Fallkonstellationen im Rahmen der Gesetzesanwendung, kann sich der anwendungsorientierte Norminhalt - «from now on»ändern. Ohne diese Neubewertung, die naturgemäß eine Auseinandersetzung mit den so far bestehenden relevanten Entscheidungen erfordert, steht jedoch keine von den bestehenden Fallentscheidungen abweichende Möglichkeit zur Falllösung offen. Sonst würde dem Charakter des Gesetzes als generelle Norm nicht entsprochen. Aus einer dialektischen Sichtweise sind (wie schon mit JOACHIM LEGE angesprochen) neue Fälle der Angelpunkt für eine Neubewertung der bestehenden Entscheidungspraxis. Diese kann den Inhalt des Gesetzes für zukünftig Entscheidungen nur solange repräsentieren, als neue Fälle im Verhältnis zu den schon entschiedenen weder wesentliche rechtliche

11 MitTERER, Das Jenseits der Philosophie (2011) etwa 58 ff; vgl. auch MitTERER, Die Flucht aus der Beliebigkeit (2011) sowie dazu Riegler/WEBER (Hrsg), Die Dritte Philosophie. Kritische Beiträge zu Josef Mitterers Non-Dualismus² (2011). 
noch sachverhaltsmäßige oder argumentative Neuerungen aufweisen. Liegen solche Neuerungen vor, kann für die nun zu treffende Entscheidung nicht ohne weiteres die bestehende Entscheidungspraxis als ident mit dem Gesetzesinhalt angenommen werden.

Bei der Entscheidung, die zu treffen ist, steht daher nicht nur die konkrete Fallkonstellation, sondern auch die Frage an, ob das Gesetz über die bestehende Falllösungspraxis hinausgehend auszulegen ist. Dann müsste der Gesetzesinhalt für den vorliegenden und für zukünftige Fälle bedeutsam - from now on - inhaltlich neu erschlossen werden. Diese Vorgabenkomponente für zukünftige Fälle übersteigt die Bedeutung der Falllösung für die konkrete Fallkonstellation.

Kontrastiert man damit die Doktrin von Montesquieu, die den Richter bloß als bouche qui prononce les paroles de la loi, als bloßen Mund des Gesetzes, erfasst ${ }^{13}$, erscheint diese Doktrin zu kurz zu greifen. Montesquieu lässt die Gesetzesanwender - kurz: die Richter - weitgehend hinter das Gesetz zurücktreten, wenn sie einen Fall nach dem Gesetz entscheiden; die Richter findet nach diesem Konzept das vorgegebene Recht und spricht es aus, ohne eine Entscheidung zu treffen. In seiner Tendenz, die Rechtsanwendung als Umsetzung normativer Vorgaben zu sehen, liegt Montesquieu aber richtig. Der Umgang mit Falllösungslinien gestaltet sich als vorgabenorientiert ${ }^{14}$. Bei der konkreten Falllösung wird auch die relevante bestehende Judikatur-Linie am Gesetz gemessen. Die Falllösung beinhaltet dann auch die Entscheidung darüber, ob diese Linie - from now on - neu ausgerichtet werden muss. Eine Neuausrichtung muss freilich ihrerseits wiederum im Gesetz gedeckt sein. Ausgehend davon repräsentieren Gesetzesrecht und darauf aufbauendes Fallrecht ein in zwei Ebenen strukturiertes System. Die beiden Ebenen interagieren immer dann, wenn ein neuer Fall zu lösen ist, wobei der Inhalt des Fallrechts letztlich am Gesetzesinhalt zu messen ist ${ }^{15}$.

MonTESQUiEu, De l'esprit des lois, Livre XI, Chapitre 6 (erstmals erschienen 1748), in: Oevres complètes, II (1951) 404.

HANDSTANGER, Zur Perspektive der Rechtsanwendung, in: Schweighofer/GeIST/HeIndL/SzÜCS (Hrsg), Komplexitätsgrenzen der Rechtsinformatik - IRIS 2008 (2008) 493. 


\section{E. KONTINUITÄT UND DISKONTINUITÄT}

Moderne Rechtsordnungen sind Mehrebenensysteme. Im Sinn eines Stufenbaus der Rechtsordnung folgt nachrangiges Recht aus höherrangigem Recht. So müssen die Gesetze den Vorgaben der vorrangigen Verfassung folgen. Gerade die im Verfassungsrang stehenden Grundrechte tragen dazu bei, dass sich die Anwendung einer Rechtsnorm diskontinuierlich gestalten kann. Neue grundrechtliche Überlegungen etwa führen zu geänderten Vorgaben, an die sich die Auslegung anpasst ${ }^{16}$. Gleiches gilt etwa für die sich aus der Beachtung des Rechts der Europäischen Union ergebenden Auswirkungen auf die nationale Rechtsordnung ${ }^{17}$.

Ob, wann, wie und wieweit eine solche Änderung auf einer höherrangigen Normebene zum Tragen kommt, hängt von einer Reihe (meist Vielzahl) von Faktoren ab, was prinzipiell durch Zusammenschau im Weg einer «Gesamtbeurteilung» evaluiert wird. In diesem Zusammenhang werden häufig nicht nur neue Argumente, sondern auch neue Argumentationskonzepte entwickelt.

Auf diesem Weg ergeben sich Diskontinuitäten bzw. Brüche, die sich zumeist nicht präzise vorhersehen bzw. vorhersagen lassen. Sind Anwendungspraxis und Normverständnis bislang kontinuierlich fortgeführt worden, so ändern sich diese dann, wenn die Gesamtbeurteilung zum Anlass für eine Diskontinuität genommen wird. Einer bislang mit einer nachvollziehbaren Kontinuitätsvermutung normativ geleitete Praxis wird dadurch disruptiv konterkariert.

Es erscheint nun fraglich, ob der Disruptionspunkt tatsächlich aus der bisherigen Praxis selbst in Verbindung mit Hinweisen auf notwendige Änderungen zeitlich und sachlich präzise vorhergesehen werden kann. Zu vielfältig und komplex sind meist die normativ vorgegebenen Gesichtspunkte, die in diesem Zusammenhang eine Rolle

Erlauben die Auslegungsstandards keine Anpassung, kommt es in aller Regel zu einer Derogation der gesetzlichen Norm.

Dies betrifft v.a. die Autonomie und den Anwendungsvorrang unionsrechtlicher Regelungen, vgl. idZ etwa EuGH (Plenum) 30.4.2019, GA 1/17, Rz 106 ff; Vgl VwGH 10.10.2018, Ra 2017/03/0108, mwH; VwGH 6.3.2019, Ro 2018/03/0031; EuGH (GK) 22.6.2010, C-188/10 ua (MELKI und ABDELI) Rz 43; EuGH 7.6.2018, C-589/16 (FILIPPI) Rz 35. 
spielen und in gegeneinander abwägenden Weise in Anschlag gebracht werden können bzw. müssen.

Ausgehend davon wird auch die Verwendung von digitalen Technologien aus dem Bereich Big Data ${ }^{18}$ bzw. von Legal Technology (Legal Tech ${ }^{19}$ ), die juristische Arbeitsprozesse unterstützen oder gänzlich automatisiert durchführen, solche Normdeutungsund anwendungsänderungen nicht präzise berücksichtigen lassen. Auf Basis solcher Technologien erarbeitete Programme, die juristische Entscheidungen (bzw. Entscheidungsalternativen) vorschlagen oder treffen sollen, könnten insofern überfordert sein.

\section{F. DIE MESOEBENE DER RECHTSSÄTZE}

Siedelt man den einzelnen Fall auf der Mikroebene und das Gesetz als Grundlage zur rechtlichen Entscheidung dieses Falles auf der Makroebne an, bestehen - intermediär positioniert - Rechtssätze aus früheren Gesetzesanwendungsfällen ${ }^{20}$. Sie beinhalten die Kernaussagen früherer juristischen Entscheidungen und stellen als Mesoebene klar, dass bestimmte Sachverhaltskonstellationen unter die Makroebene Gesetz fallen. Kooperativ in rechtlichen Verfahren von Gerichten und Parteien entwickelt stabilisieren sie die Gesetzesanwendung in zur Entscheidung anstehenden neuen Fällen. Gerade die Rechtssätze, die die ständige Rechtsprechung von gerichtlichen Grenzorganen verkörpern, zeigen maßgeblich den Inhalt von Gesetzen auf.

Ein auf einem Begriffsthesaurus aufbauenden rechtsinformatischen System wie das RIS erlaubt derzeit (jedenfalls für die Praxis) gute Suchergebnisse im Sinn der Unterstützung juristischer Tätigkeit durch Produkte der Rechtsinformatik. Im RIS werden die gerichtlichen Entscheidungen in dem gesamten Text wiedergegeben, ebenso die in der Entscheidung identifizierten Rechtssätze. Die Rechtssätze (ebenso wie der Entscheidungstexte selbst) verfügen über Metadaten, eingegeben werden dort neben den bezogenen Gesetzesstellen insbesondere für den Rechtssatz zentrale Begriffe. Ferner 
lässt sich eine Verknüpfung inhaltlich gleichartiger Rechtssätze herstellen («Rechtssatzkette»). Der Metatext erlaubt eine Beschlagwortung nach Worten, die nicht im Rechtssatz selbst aufscheinen. Außerdem lässt sich etwa anmerken, in welcher Entscheidung der Rechtssatz das erste Mal vorkommt und insofern die Grundlage für die Rechtssatzkette identifizieren. Sucht man in der Menge der Rechtssätze nach einem bestimmten Begriff «X», erhält man als Suchergebnis die Rechtssätze, in denen der Begriff entweder im Rechtssatztext selbst oder in den Metadaten des Rechtsatzes aufscheint.

Für die Unterstützung zur leichteren Produktion von wäre es aber zu kurz gegriffen, wenn die entscheidende Stelle lediglich auf die Suche mittels Begriffen aufbauen müsste. Vielmehr erfordert ein formulierungsunterstützendes rechtsinformatisches System wohl, dass sich die Suche nicht auf Begriffe beschränkt, sondern ganze Terme als sinnzusammenhängende Wortgruppe erfasst. Würde der Metatext des Rechtssatzes sowie der dahinter stehende Thesaurus um solche Terme ergänzt, könnte es eine termorientierte Verzettelung der Rechtssätze erlauben, die in den Rechtssätze schon enthaltenen Terme (ganze Wortgruppen oder auch Sätze) bei entsprechender Suche direkt in den Entscheidungstext einzubauen. Im Ergebnis würde damit die Bedeutung solcher Rechtssätze für die Entscheidungsfindung und Entscheidungstextierung noch erhöht. Die termorientierte Aufbereitung von Rechtssätzen geht in die Richtung einer Fusion der derzeit auf Begriffssuche ausgerichteten Arbeitsmethode mit Ansätzen zur automatischen Texterstellung. Diese Fusion könnte einen wesentlichen weiteren Schritt für die Entwicklung der rechtsinformatikgestützten Entscheidungstexterstellung bedeuten. Bedacht zu nehmen wäre freilich darauf, dass die Formulierung von Termen ihrerseits im Kontext der konkret zu treffenden Entscheidungen bzw. der darin zum Ausdruck kommenden vielfältigen Bezüge steht. Gerade die termgestützte Rechtssatztechnik ermöglicht aber die Abbildung ganz unterschiedlicher Formulierungen (als Terme) für jeweils bestimmte Fragen. Mit dieser Technik lässt sich die Diversifikation gegenüber einer Verknappung von Formulierungen und damit letztlich auch von Lösungen favorisieren und gleichzeitig die breitere Verwendung der so auffindbaren Rechtssätze wesentlich erleichtern. Die termbezogene Verzettelung nutzt die Formulierungsgewohnheiten der Praxis, die grundsätzlich in die Richtung weisen, dass gleiche Phänomene in den Entscheidungstexten immer wieder mit gleichartigen Formulierungen Berücksichtigung finden. 
